\title{
Force acting on a square cylinder fixed in a free-surface channel flow
}

\author{
Z. X. Qi ${ }^{1}, I$ Eames ${ }^{1} \dagger$ and E. R. Johnson ${ }^{1}$ \\ ${ }^{1}$ University College London, Torrington Place, London WC1E 7JE, UK
}

(Received 22 October 2013; revised 9 June 2014; accepted 1 August 2014; first published online 4 September 2014)

\begin{abstract}
We describe an experimental study of the forces acting on a square cylinder (of width $b$ ) which occupies $10-40 \%$ of a channel (of width $w$ ), fixed in a free-surface channel flow. The force experienced by the obstacle depends critically on the Froude number upstream of the obstacle, $F r_{1}$ (depth $h_{1}$ ), which sets the downstream Froude number, $F r_{2}$ (depth $h_{2}$ ). When $F r_{1}<F r_{1 c}$, where $F r_{1 c}$ is a critical Froude number, the flow is subcritical upstream and downstream of the obstacle. The drag effect tends to decrease or increase the water depth downstream or upstream of the obstacle, respectively. The force is form drag caused by an attached wake and scales as $\overline{F_{D}} \simeq C_{D} \rho b u_{1}^{2} h_{1} / 2$, where $C_{D}$ is a drag coefficient and $u_{1}$ is the upstream flow speed. The empirically determined drag coefficient is strongly influenced by blocking, and its variation follows the trend $C_{D}=C_{D 0}\left(1+C_{D 0} b / 2 w\right)^{2}$, where $C_{D 0}=1.9$ corresponds to the drag coefficient of a square cylinder in an unblocked turbulent flow. The r.m.s. lift force is approximately $10-40 \%$ of the mean drag force and is generated by vortex shedding from the obstacle. When $\operatorname{Fr}_{1}=\operatorname{Fr}_{1 c}(<1)$, the flow is choked and adjusts by generating a hydraulic jump downstream of the obstacle. The drag force scales as $\bar{F}_{D} \simeq C_{K} \rho b g\left(h_{1}^{2}-h_{2}^{2}\right) / 2$, where experimentally we find $C_{K} \simeq 1$. The r.m.s. lift force is significantly smaller than the mean drag force. A consistent model is developed to explain the transitional behaviour by using a semi-empirical form of the drag force that combines form and hydrostatic components. The mean drag force scales as $\overline{F_{D}} \simeq \lambda \rho b g^{1 / 3} u_{1}^{4 / 3} h_{1}^{4 / 3}$, where $\lambda$ is a function of $b / w$ and $F r_{1}$. For a choked flow, $\lambda=\lambda_{c}$ is a function of blocking $(b / w)$. For small blocking fractions, $\lambda_{c}=C_{D 0} / 2$. In the choked flow regime, the largest contribution to the total drag force comes from the form-drag component.
\end{abstract}

Key words: channel flow, free-surface flow, hydraulics

\section{Introduction}

The Japan (2011) and Indian Ocean (2004) tsunami led to significant loss of life and destruction of city infrastructure, including businesses and homes. Building failure occurred due to a combination of earthquake damage and inundation by sea water as the tsunami moved inland. While the initial damage in coastal regions was largely 
caused by slamming forces, significant damage was caused by the quasi-steady flow of sea water around buildings, which generated stress on the building panels, torque on the buildings and buoyancy forces that carried wooden buildings away. Part of the challenge in designing buildings that can resist these forces is in gaining a better understanding of the physics and conservative estimates of the total force acting on these structures.

Research on obstacles in free-surface channel flows is very well established. Early theoretical studies by Nagler (1918) and the large-scale experiments of Yarnell $(1934 a, b)$ focused on predicting changes in the upstream water depth - the backwater effect - caused by streamlined piers. The key concept these authors introduced was a classification of the flow types observed, which we describe here as being either subcritical or choked. For a subcritical flow, the change between upstream and downstream water depth is small and the flow is essentially around the obstacle. For a choked flow, the change in water depth is quite dramatic and is characterised by a hydraulic jump downstream.

This paper focuses on the force experienced by a rigid obstacle, which in this case is taken to be a square cylinder, a shape that is representative of a building. Most studies have focused on the subcritical regime (Azinfar \& Kells 2009), which is a typical situation in civil engineering because the hydraulic jump in the choked regime promotes scour, for example near the foundations of a bridge. Earlier work by Raju et al. (1983) analysed the drag force on circular cylindrical structures in the subcritical regime; the forces were determined by integrating the pressure distribution over the whole surface of the cylinder. The one-dimensional model developed accounted for form drag, and experimental results were compared against it. The analysis of Raju et al. (1983) was made tractable by assuming that the change in water depth is small; such assumptions are not appropriate for choked flows. Most other studies have estimated drag forces from the loss of momentum flux of the current stream, using measurements of average water depth (e.g. Henderson 1966). This technique cannot be used for lift forces and is difficult to apply to three-dimensional bodies because the wave field is unsteady.

In this paper we describe a detailed experimental study of the lift and drag forces acting on a square obstacle, over a range of blocking fractions. In $\S 2$, we describe a one-dimensional model which is closed using a semi-empirical form for the drag force, aspects of which have been briefly analysed by Fenton (2003). The purpose of the present work is to determine the empirical drag and hydrostatic coefficients, particularly in the choked flow regime. In $\S 3$, the experimental methodology is described, where an obstacle is mounted on an instrumented drag/lift cell. The results are described and compared with the one-dimensional theory in $\S 4$, where we show consistency of a priori predictions for the inferred drag and hydrostatic coefficients with observed changes to the free surface. Finally, conclusions are drawn in $\S 5$.

\section{Mathematical model}

We develop a one-dimensional model with a semi-empirical form for the force closure to understand the link between the force and changes in the free-surface shape. Figure 1 shows a schematic of the problem, which consists of a flow in a rectangular channel characterised by a water depth $h$ and average speed $u$. The channel width far upstream and downstream of the square obstacle (of width $b$ ) is $w$, and the flow passes a constriction where the width decreases to a minimum of $w-b$. The volume flux $Q$ is conserved, so that upstream and downstream of the obstacle,

$$
Q=u w h .
$$




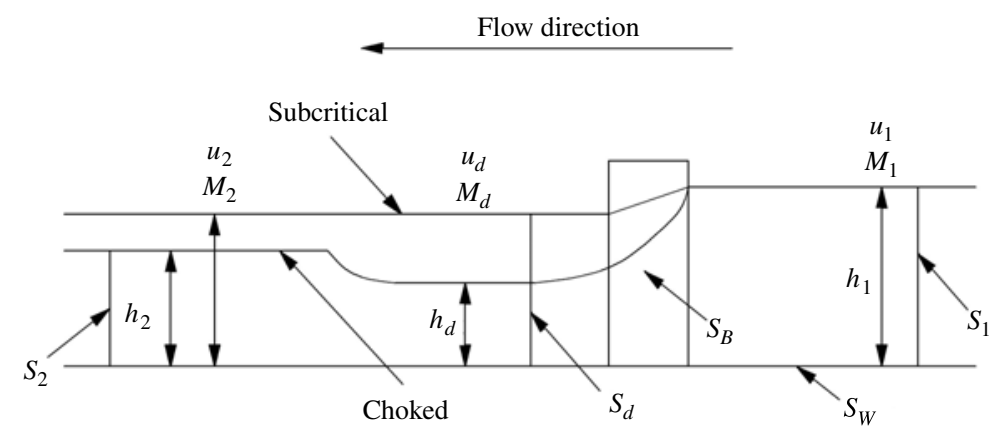

FIGURE 1. Schematic of the side view of a free-surface channel flow around a fixed surface-piercing obstacle. Here $S_{1}, S_{d}, S_{B}$ and $S_{W}$ are as defined in the text, and $S_{2}$ is the control surface far downstream of the obstacle.

The sum of the specific momentum flux and specific hydrostatic force on a vertical plane, denoted by $M$, and the Froude number, $F r$, are defined by

$$
M=\left(u^{2} h+\frac{1}{2} g h^{2}\right) w, \quad F r=\frac{u}{\sqrt{g h}} .
$$

Since $Q$ is invariant, when we consider integral measures across the channel width $w$ we can express $h$ and $M$ in terms of $Q$ and $F r$ as follows:

$$
h=\frac{Q^{2 / 3}}{w^{2 / 3} F r^{2 / 3} g^{1 / 3}}, \quad M=\frac{g^{1 / 3} Q^{4 / 3}}{w^{1 / 3} F r^{4 / 3}}\left(\frac{1}{2}+F r^{2}\right) .
$$

Integrating the steady Navier-Stokes equation over the flow domain gives

$$
\int_{S_{B}}(p \hat{\boldsymbol{n}}-\boldsymbol{\tau} \cdot \hat{\boldsymbol{n}}) \mathrm{d} S=-\int_{S_{1}+S_{d}} p \hat{\boldsymbol{n}} \mathrm{d} S-\rho \int_{S_{1}+S_{d}}(\boldsymbol{u} \cdot \hat{\boldsymbol{n}}) \boldsymbol{u} \mathrm{d} S+\int_{S_{1}+S_{d}+S_{W}} \boldsymbol{\tau} \cdot \hat{\boldsymbol{n}} \mathrm{d} S,
$$

where $\hat{\boldsymbol{n}}$ is the unit normal directed out of the fluid domain, $p$ is the pressure (including the hydrostatic component) and $\tau$ is the viscous stress tensor. Here $S_{1}$ and $S_{d}$ are the control surfaces upstream and immediately downstream of the obstacle, $S_{B}$ is the wetted surface of the obstacle and $S_{W}$ is the wetted surface of the channel walls and floor. The left-hand side of (2.4) is the force on the obstacle, $\boldsymbol{F}$ (e.g. Nicolle \& Eames 2011). Provided that spatial gradients in the flow far upstream and downstream are weak, the viscous stresses in the last term of (2.4) are negligible. We resolve the momentum (2.4) parallel to the incident stream, where the drag on the obstacle is

$$
\overline{F_{D}}=\overline{\boldsymbol{F}} \cdot \hat{\boldsymbol{x}}=\frac{1}{2} \rho g\left(h_{1}^{2}-h_{d}^{2}\right) w+\rho\left(u_{1}^{2} h_{1}-u_{d}^{2} h_{d}\right) w=\rho\left(M_{1}-M_{d}\right),
$$

with

$$
\boldsymbol{F}=\int(p \hat{\boldsymbol{n}}-\boldsymbol{\tau} \cdot \hat{\boldsymbol{n}}) \mathrm{d} S
$$

This integral relationship between $\overline{F_{D}}$ and $M_{1}$ and $M_{d}$ is well known (e.g. Benjamin 1956, equation (1.3); Massey \& Ward-Smith 1998). The presence of a rigid body, 
introduced into a channel flow, generates a perturbation. At low Fr, the change in water depth upstream and downstream is negligible. But at high Fr, a bore initially propagates upstream and downstream from the body when the water is set in motion, leading to a change in the water depth upstream and downstream of the obstacle; this change in water depth may be dramatic. This means that a reference, unperturbed upstream state will quickly be lost, especially in practice and in our experiments within a flume of finite length. For this reason, the model and observations are expressed in terms of an upstream Froude number, $F r_{1}$. Given an upstream state (characterised by $F r_{1}$ ), the purpose is to determine the downstream state characterised by $\mathrm{Fr}_{2}$ and the mean drag force on the obstacle.

There are a number of closures that could be applied to estimate the state of the flow, and these involve making assumptions about how energy or momentum is conserved across the throat of the flow. The momentum approach is the one which has been favoured since the 1960 s and is conceptually the closest to describing the action of the obstacle. Fenton $(2003,2008)$ provided a clear description of these processes, mostly in the limit of small changes in the water depth, whereas the current study focuses on the nonlinear effects. The basis of the empirical closure is a mean drag force, estimated by adding together a form drag, based on the wetted frontal area of the obstacle $(b h)$ and the mean flow $u$ (area-averaged), and the hydrostatic force, so that

$$
\overline{F_{D}}=\frac{1}{2} C_{D} \rho b u^{2} h+\frac{1}{2} C_{H} \rho b g\left(h_{1}^{2}-h_{d}^{2}\right),
$$

where $C_{D}$ and $C_{H}$ are empirically determined constants. The left-hand side of (2.7) is equal to the right-hand side of (2.5). Henderson (1966) and Fenton (2003), for instance, included only the first form-drag term, while Fenton (2008) included both terms (although the second term far downstream was expressed in terms of $h_{2}$ ). The usual approach is to prescribe $C_{D}$ based on studies where obstacles are submerged in a channel flow. The blocking caused by the obstacle has a significant influence on the drag force. Given the drag coefficient $C_{D 0}$ for an unbounded flow, we can estimate the influence of blocking. For an unbounded flow $u$, the drag on a body at high Reynolds numbers generates a volume flux $Q_{w}=C_{D 0}$ buh downstream in an unblocked flow (Betz 1925). The effect of blocking increases the average flow outside the wake. The obstacle sees an average of the upstream flow $u$ and the downstream flow $u+Q_{w} / h w$, i.e. $u\left(1+C_{D 0} b / 2 w\right)$, generating a drag force of $C_{D 0} b u^{2}\left(1+C_{D 0} b / 2 w\right)^{2} / 2$, which is equivalent to a drag coefficient of

$$
C_{D}=C_{D 0}\left(1+\frac{C_{D 0} b}{2 w}\right)^{2}
$$

(For circular cylinders, Raju et al. 1983 used an empirical form $C_{D}=C_{D 0}(1-$ $b / w)^{-1.35}$, whereas Maskel 1963 and Awbi 1978 used $C_{D}=C_{D 0}\left(1+\eta C_{D 0} b / w\right)$, where $\eta$ is a blockage factor dependent on the magnitude of the base-pressure coefficient.) The drag coefficient for square cylinders is influenced by ambient turbulence; for a turbulent intensity of $5 \%$, which is typical of the conditions of the experimental flume described in $\S 3, C_{D 0}$ drops from 2.1 to 1.9 (Tamura \& Miyagi 1999). There are various ways to parameterise $h$ and $u$; one is to take $h=h_{1}$ and $u=Q / h_{1} w$, and this is the choice adopted here. Recasting (2.7) in a dimensionless form, we obtain 
an implicit relationship between $F r_{1}$ and $F r_{d}$,

$$
\left(1-\frac{C_{H} b}{w}\right) \frac{1}{2 F r_{1}^{4 / 3}}+\left(1-\frac{C_{D} b}{2 w}\right) F r_{1}^{2 / 3}=\left(1-\frac{C_{H} b}{w}\right) \frac{1}{2 F r_{d}^{4 / 3}}+F r_{d}^{2 / 3} .
$$

Equation (2.9) is solved with $F r_{d}$ as a function of $F r_{1}$ to relate the state of the downstream flow to that of the upstream flow. This yields a maximum value of $F r_{1 c}$ which corresponds to $\partial F r_{1} / \partial F r_{d}=0$. Differentiating (2.9), we have

$$
F r_{d c}=\left(1-\frac{C_{H} b}{w}\right)^{1 / 2}
$$

Under this formulation, $F r_{d c}$ depends only on $C_{H} b / w$.

The transition from $S_{d}$ to $S_{2}$ (the control surface far downstream of the obstacle) is determined by the condition that (in the absence of friction) the momentum flux is conserved, i.e.

$$
\frac{1}{F r_{d}^{4 / 3}}\left(\frac{1}{2}+F r_{d}^{2}\right)=\frac{1}{F r_{2}^{4 / 3}}\left(\frac{1}{2}+F r_{2}^{2}\right)
$$

which yields either $F r_{2}=F r_{d}$ for a subcritical throat flow or $F r_{2}=F r_{2 c}\left(>F r_{d}\right)$ for a supercritical throat flow. Here $F r_{1 c}$ and $F r_{2 c}$ are determined from (2.9) and (2.11), respectively.

\section{Experimental set-up and methodology}

The experiments were undertaken in a flume of width $0.50 \mathrm{~m}$, depth $0.20 \mathrm{~m}$ and length $3 \mathrm{~m}$, whose inlet is tapered to minimise upstream disturbance. The volume flux through the flume was estimated from a time-averaged measurement of velocity using an acoustic doppler velocimeter (Nortek Vectrino Ltd). The turbulent intensity, $I_{t}=u_{r m s} / \bar{u}$ (where $u_{r m s}$ is the r.m.s. velocity and $\bar{u}$ the time-averaged velocity), was measured as $I_{t}=5.5 \%$. A number of square acrylic blocks (of widths $b=0.05,0.10$, 0.15 and $0.20 \mathrm{~m}$ and of height $0.20 \mathrm{~m}$ ) were mounted onto a rigid rod, connected to two calibrated load cells. The voltage outputs from the local Wheatstone bridges varied linearly with the applied moments and were logged with two DSC USB digitisers. Both the drag $\left(F_{D}\right)$ and lift $\left(F_{L}\right)$ forces may vary in time. These are characterised in terms of the mean drag and r.m.s. lift forces, defined as

$$
\overline{F_{D}}=\frac{1}{T} \int_{0}^{T} F_{D} \mathrm{~d} t, \quad F_{L, r m s}=\left(\frac{1}{T} \int_{0}^{T} F_{L}^{2} \mathrm{~d} t\right)^{1 / 2} .
$$

The r.m.s. lift force is used because the average lift force is zero, i.e. $(1 / T) \int_{0}^{T} F_{L} \mathrm{~d} t=$ 0 . The water depth upstream and downstream of the rectangular obstacle was measured directly from the sides of the tank far downstream of the hydraulic jump.

\section{Experimental results}

To examine the influence of the channel flow on the force acting on the obstacle, both the channel volume flux $Q$ and the initial channel water depth $h_{I}$ were varied. The initial water depth $h_{I}$ was varied from 0.02 to $0.18 \mathrm{~m}$ (in increments of $0.02 \mathrm{~m}$ ), and $Q$ varied from 0.013 to $0.065 \mathrm{~m}^{3} \mathrm{~s}^{-1}$ (in increments of $0.013 \mathrm{~m}^{3} \mathrm{~s}^{-1}$ ). For the shallowest downstream water depths, frictional effects appear to affect the results, and therefore the results for $h_{I} \leqslant 0.06 \mathrm{~m}$ were neglected in the data collation. 

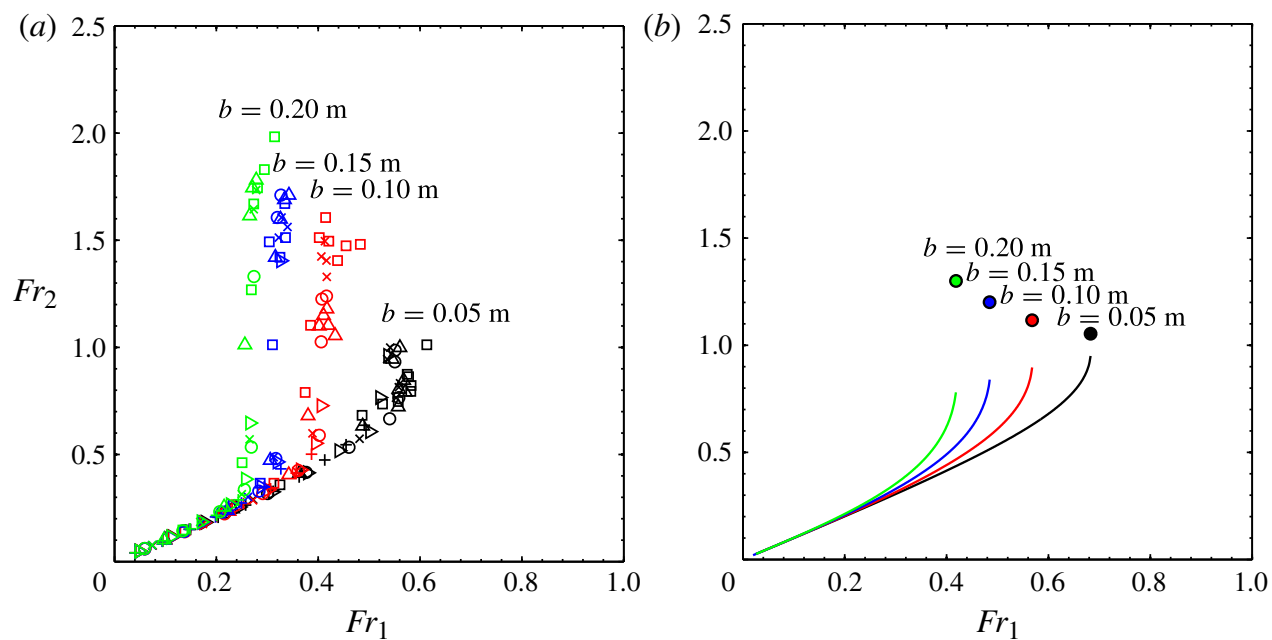

FIgURE 2. (Colour online) Scatter plots of $F r_{2}$ versus $F r_{1}$ (defined by (2.2)) for an obstacle of width $b=0.05,0.10,0.15$ or $0.20 \mathrm{~m}$ : $(a)$ experimental; $(b)$ model-predicted. The symbols in panel $(a)$ correspond to $h_{I}=0.08(\square), 0.10(\triangle), 0.12(\times), 0.14(\mathrm{O})$, $0.16(\triangleright)$ and $0.18(+) \mathrm{m}$. The curves in panel $(b)$ represent $(2.9)$ with $C_{D 0}=1.9$ and $C_{H}=0.58$, and the filled circles correspond to choked flow.

\subsection{Water depth}

Figure 2 shows scatter plots of the experimental and model-predicted upstream and downstream Froude numbers. The data tend to fall into two regions, as discussed in the introduction and by Brocchini \& Peregrine (2001). Below a subcritical upstream Froude number, i.e. $F r_{1}<F r_{1 c}, F r_{1}$ and $F r_{2}$ are correlated with $F r_{2} / F r_{1} \approx 1$, such that the difference between the upstream and downstream depths is small. This is reflected in the forms of surface disturbance seen in figures 3(a) and 4(a). The small-amplitude undulations observed are capillary waves whose form is fixed by the flow around the obstacle or which are shed from its corners. The free surface is slightly deformed by the wake shedding and is turbulent, as is evident by the roughening of the free surface. At $F r_{1}=F r_{1 c}$, the downstream Froude number jumps to $F r_{2 c}$. These changes are clearly observed in the nature of the free-surface disturbance. As the Froude number $F r_{1}$ approaches a critical value, we observe that the water depth decreases rapidly around the sides of the obstacle and a hydraulic jump is generated just downstream (see figures $3 b, c$ and $4 b, c$ ). In this region the water is shallow and recovers slightly further downstream. Also evident is that the flow is clearly not one-dimensional. The model is based on a one-dimensional analysis that ignores sidewall and floor friction. From figure 4, we can see a standing wave field and the hydraulic jump downstream of the square cylinder. The difference between the simple model and the observations occurs in the estimation of $\mathrm{Fr}_{2}$, and this is due to the challenge of measuring the water depth and the influence of the wave.

\subsection{Force on the obstacle}

Figure 5 shows a typical time series for the drag and lift force coefficients of a square cylinder (with $b / w=0.2$ ) in the subcritical and choked regimes. For the 
(a)
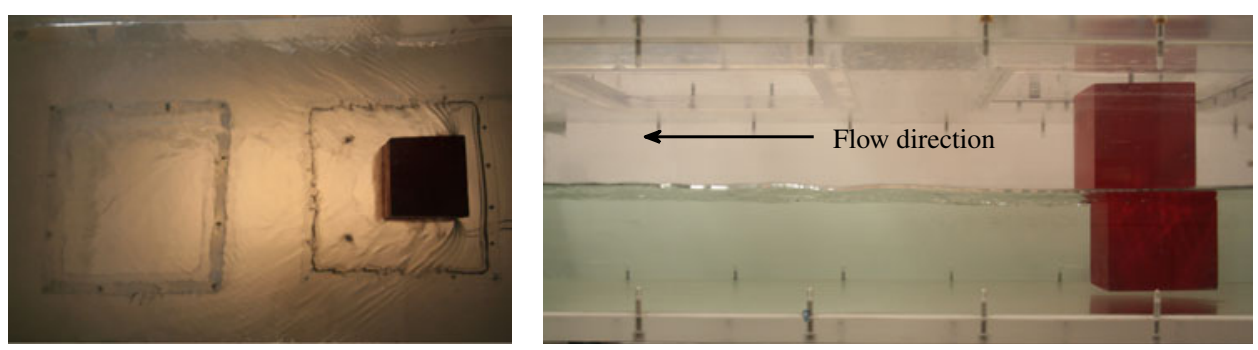

(b)
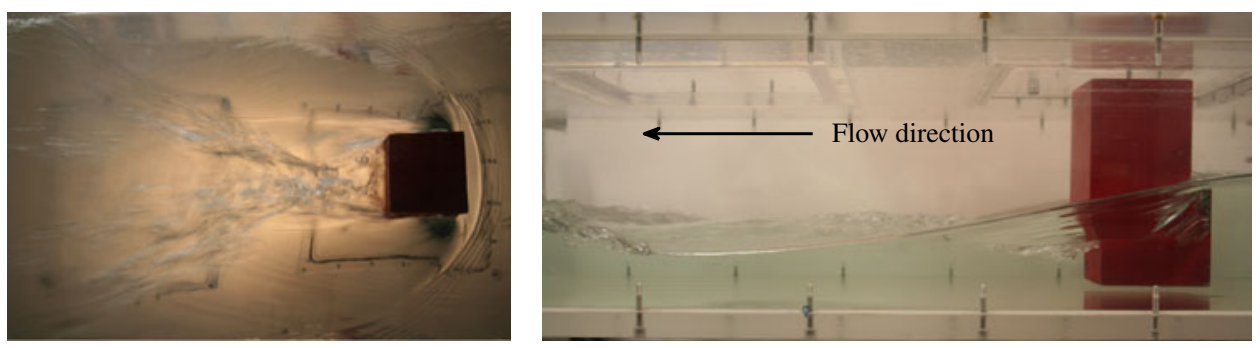

(c)
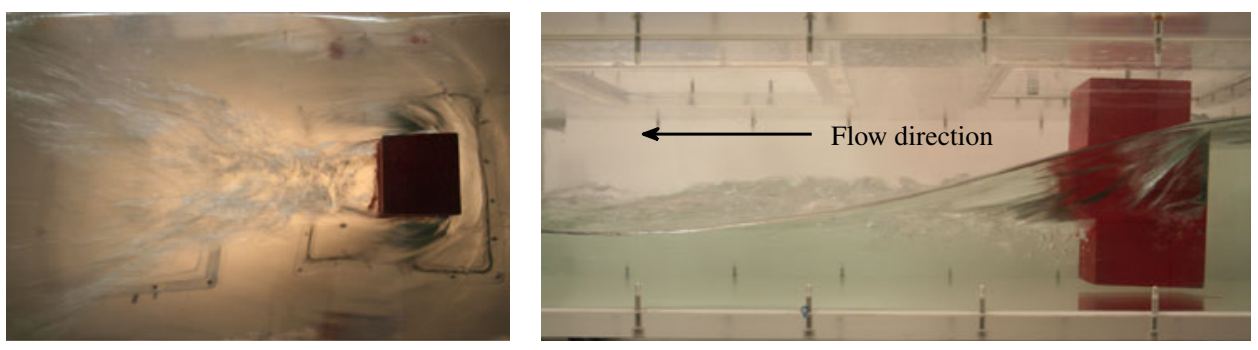

FIGURE 3. Photographs of the free-surface shape for fixed water depth $\left(h_{I}=0.10 \mathrm{~m}\right)$, fixed obstacle width $(b=0.10 \mathrm{~m})$ and increasing flow rates. The flow is from right to left. In each panel the plan view is shown on the left and the side view is shown on the right. The images correspond to: (a) $F r_{1}=0.13, F r_{2}=0.13 ;$ (b) $F r_{1}=0.37, F r_{2}=0.46$; (c) $F r_{1}=0.42, F r_{2}=1.22$.

subcritical regime (see figure $5 a$ ), the r.m.s. lift coefficient is $10-40 \%$ of the mean drag coefficient. As the flow rate increases, the frequency of shedding increases. In the choked region, the fluctuating lift force is negligible compared to the drag force (see figure $5 b$ ).

Figure 6(a) shows the variation of $2 \overline{F_{D}} / \rho b u_{1}^{2} h_{1}$ with $F r_{1}$. For $F r_{1}<F r_{1 c}$, we observe that the drag coefficient is approximately constant, but as the controlled state is approached, the data do not collapse but increase significantly. The hydrostatic balance is more appropriate in the choked flows, $F r_{1} \simeq F r_{1 c}$. Figure $6(b)$ shows the variation of $2 \overline{F_{D}} / \rho b g\left(h_{1}^{2}-h_{2}^{2}\right)$ with $F r_{1}$. The results tend to suggest a constant coefficient in the choked regime. The r.m.s. lift coefficient is plotted in figure $6(d)$, and comparison with figure 6(a) shows that the r.m.s. lift force is at most $40 \%$ of the drag force in the low-Froude-number regime and decreases significantly to less than $1 \%$ of the drag force close to and at the choked regime. In the choked regime, there is a significant height difference between the upstream and downstream regions. The fraction of the wetted region on the downstream side of the square cylinder in the choked regime is approximately $30 \%$ of the upstream wetted area (see side-view images on the right in figure $3 b, c)$. Since $C_{D} \approx 4$ in the choked regime, we would 
(a)

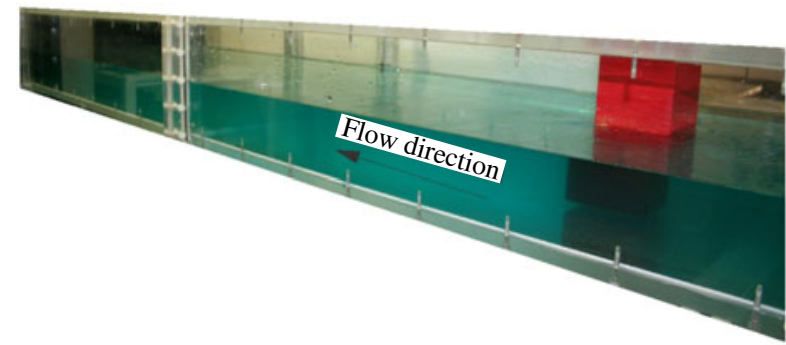

(b)

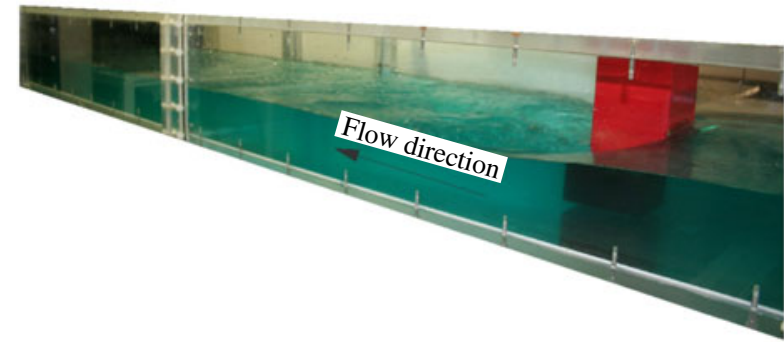

(c)

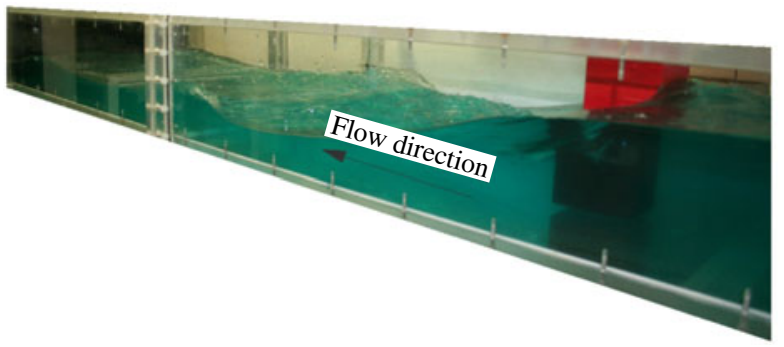

FIGURE 4. Photographs of the free-surface shape from an elevated side view for fixed water depth $\left(h_{I}=0.10 \mathrm{~m}\right)$, fixed obstacle width $(b=0.10 \mathrm{~m})$ and increasing flow rates. The flow is from right to left. The images correspond to: $(a) F r_{1}=0.13, F r_{2}=0.13$; (b) $F r_{1}=0.37, F r_{2}=0.46 ;$ (c) $F r_{1}=0.42, F r_{2}=1.22$.

estimate that $C_{L, r m s} \approx 0.3 \lambda C_{D}$, where $\lambda=0.8 / 2.5$. This means that $C_{L, r m s}$ is estimated to be approximately 0.4 . In fact, we find that $C_{L, r m s}$ is $0.08-0.2$ (see figure $5 d$ ), which is much less than could be explained by the reduction in the wetted area. From the plan-view images on the left of figure $3(b, c)$, we see that the flow is accelerating downstream of the cylinder because the cross-sectional area of the water is decreasing. This generates a straining flow at the rear of the cylinder, which suppresses the usual von Karman wake shedding by bringing together the two shear layers. The (turbulent) hydraulic jump forms in the near-wake region, and this is again likely to disrupt any vortex shedding. The drag coefficient $C_{D}$ is determined from the low- $F r$ experiments and is defined here as

$$
C_{D}=\lim _{F r_{1} \rightarrow 0} \frac{2 \overline{F_{D}}}{\rho b u_{1}^{2} h_{1}} .
$$

The variation of $C_{D}$ with blocking is shown in figure $7(a)$, along with the prediction (2.8) where $C_{D 0}=1.9$ corresponds to the drag on a square cylinder in an unblocked turbulent flow. The dashed curve corresponds to the prediction for a non-turbulent flow $\left(C_{D 0}=2.1\right)$ and agrees with published three-dimensional large eddy simulation 

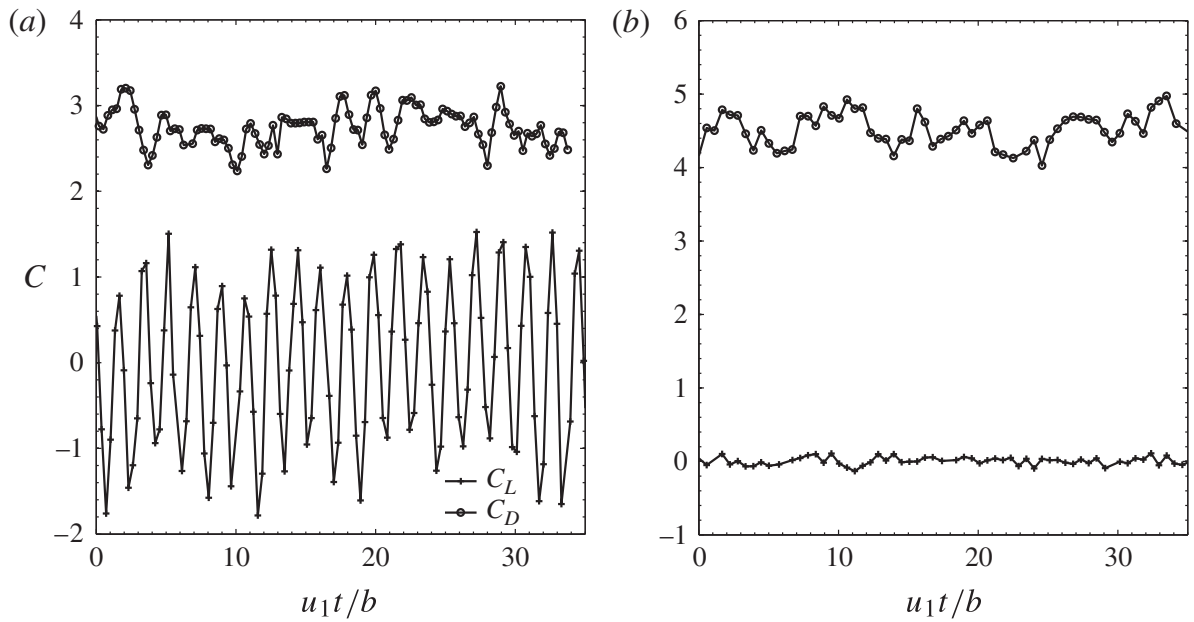

FIgURE 5. Time series of drag and lift coefficients (defined as $C=2 F / \rho b u_{1}{ }^{2} h_{1}$ ) plotted for (a) subcritical flow, $F r_{1}=0.13$, and $(b)$ choked flow, $F r_{1}=0.45$. Here $b / w=0.2$ and the initial water depth is $h_{I}=0.10 \mathrm{~m}$.

calculations. The hydrostatic coefficient, defined as

$$
C_{K}=\lim _{F r_{1} \rightarrow F r_{1 c} c} \frac{2 \overline{F_{D}}}{\rho b g\left(h_{1}^{2}-h_{2}^{2}\right)},
$$

is plotted in figure $7(b)$. The results are consistent with the expectation that $C_{K} \approx 1$. In the choked regime, a portion of the force will be still derived from form drag, and so the empirical coefficient $C_{H}$ must be inferred. From (4.2), (2.7), (2.2b) and (2.3a),

$$
C_{K}=C_{D} \frac{F r_{1 c}^{2 / 3}}{F r_{1 c}^{-4 / 3}-F r_{2 c}^{-4 / 3}}+C_{H} \frac{F r_{1 c}^{-4 / 3}-F r_{d c}^{-4 / 3}}{F r_{1 c}^{-4 / 3}-F r_{2 c}^{-4 / 3}} \text {. }
$$

The value of $C_{H}$ is estimated by using a least-relative-error fit between (4.3) and experimental measurements, using empirical values for $F r_{1 c}$ and an estimate of $F r_{d c}$, generating a value of $C_{H}=0.58$. Figure $7(b)$ shows a comparison between the $C_{K}$ determined experimentally and the prediction given by (4.3).

The problem with using (4.2) in a predictive setting is that it depends on information both upstream and downstream of the obstacle. An alternative way of bringing together the two force scalings can be identified from (2.3), giving rise to

$$
\overline{F_{D}}=\lambda \rho b g^{1 / 3} u_{1}^{4 / 3} h_{1}^{4 / 3}
$$

where

$$
\lambda=\frac{1}{2} C_{D} F r_{1}^{2 / 3}+\frac{1}{2} C_{H}\left(\frac{1}{F r_{1}^{4 / 3}}-\frac{1}{F r_{d}^{4 / 3}}\right) .
$$

Figure $6(c)$ shows the collapse of $\lambda$ onto one curve for the subcritical and choked regimes. Focusing on the choked flow regime, $\lambda=\lambda_{c}$, the variation of $\lambda_{c}$ with blocking is shown in figure $7(c)$, where the symbols correspond to the critical value of $\lambda$ in figure $6(c)$ for the choked regime. The curve in figure $7(c)$ corresponds to 

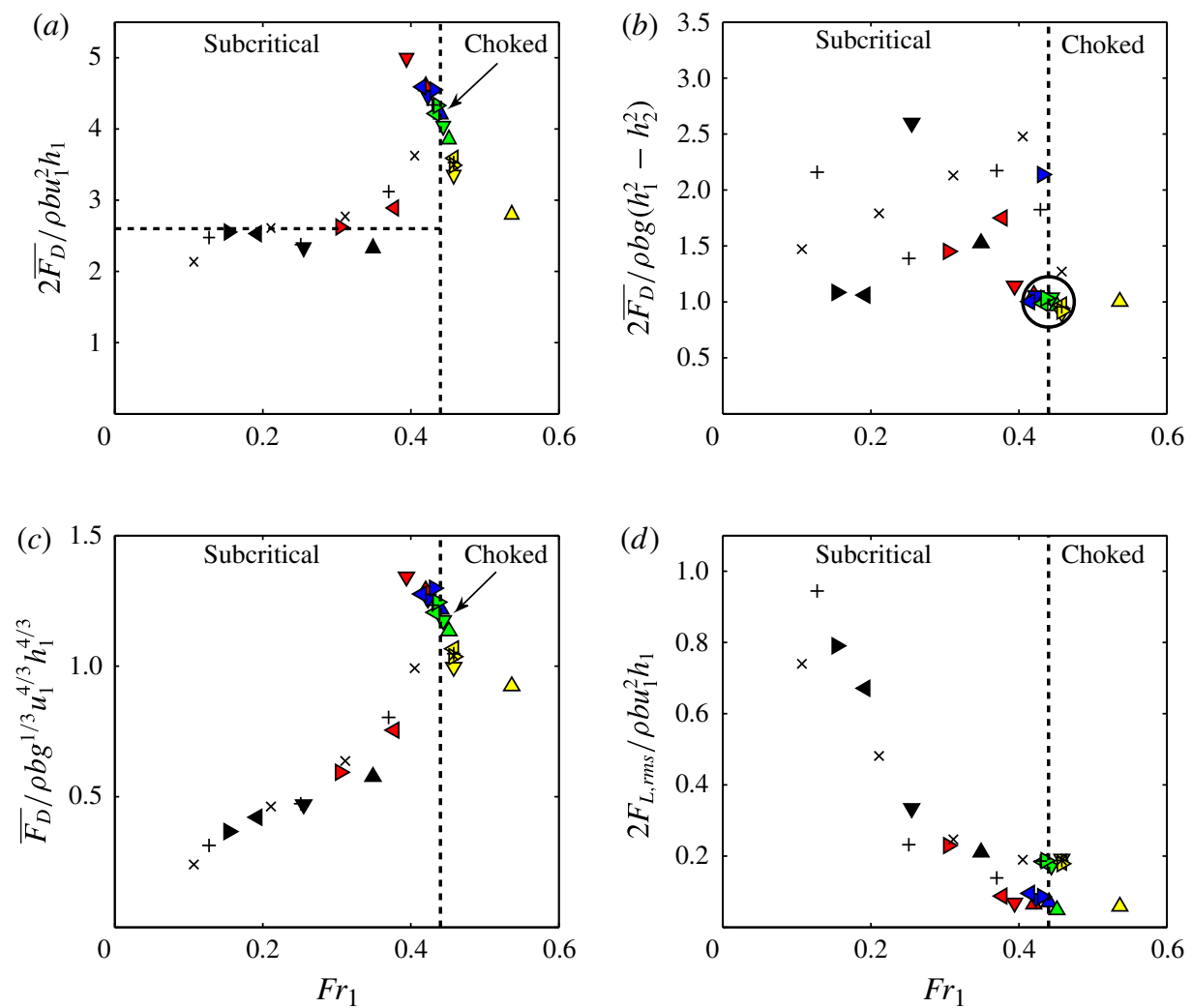

FIGURE 6. Force coefficients on the obstacle plotted as functions of $F r_{1}$, for $b / w=0.2$ : (a) drag force normalised as $2 \overline{F_{D}} / \rho b u_{1}^{2} h_{1} ;(b)$ drag force normalised as $2 \overline{F_{D}} / \rho b g\left(h_{1}^{2}-h_{2}^{2}\right)$; (c) drag force normalised as $\overline{F_{D}} / \rho b g^{1 / 3} u_{1}^{4 / 3} h_{1}^{4 / 3} ;(d)$ r.m.s. lift force $2 F_{L, r m s} / \rho b u_{1}^{2} h_{1}$. The symbols correspond to $h_{I}=0.08(\triangle), 0.10(\nabla), 0.12(\triangleleft), 0.14(\triangleright), 0.16(+)$ and $0.18(\times) \mathrm{m}$; the colours correspond to $Q=0.013$ (black), 0.026 (red), 0.039 (blue), 0.052 (green) and 0.065 (yellow) $\mathrm{m}^{3} \mathrm{~s}^{-1}$. The points for the choked flow accumulate at $F r_{1} \simeq 0.43$.

the prediction. For small blocking fractions, the critical upstream Froude number $F_{1}$ tends to 1 , and $\lambda$ is dominated by the first term in (4.5) so that $\lambda_{c}=C_{D 0} / 2$.

Having chosen $C_{H}, \lambda_{c}$ can be estimated without recourse to force measurements by using measurements of $F r_{1}$. Figure $7(d)$ plots the variation of $\lambda_{c}$ with blocking, and this shows good agreement with $\lambda_{c}$ derived from force measurements. The partition between hydrostatic and form-drag components cannot be measured but can be inferred. The form and hydrostatic components can be identified as the first and second terms on the right-hand side of (4.5). Figure $7(d)$ shows the variation of the hydrostatic $\left(\lambda_{c, H}=C_{H}\left(F r_{1 c}^{-4 / 3}-F r_{d c}^{-4 / 3}\right) / 2\right)$ and form-drag $\left(\lambda_{c, D}=C_{D} F r_{1 c}^{2 / 3} / 2\right)$ components with blocking. For low blocking fractions, the hydrostatic component is negligible, but as the blocking fraction increases, the hydrostatic contribution increases. Interestingly, the form-drag contribution is weakly affected by blocking. This is because while the drag coefficient increases with blocking (figure $7 a$ ), there is an equivalent reduction with $F r_{1 c}$, leading to a small change in the form-drag contribution. Surprisingly, the results show that while we do observe a dramatic 

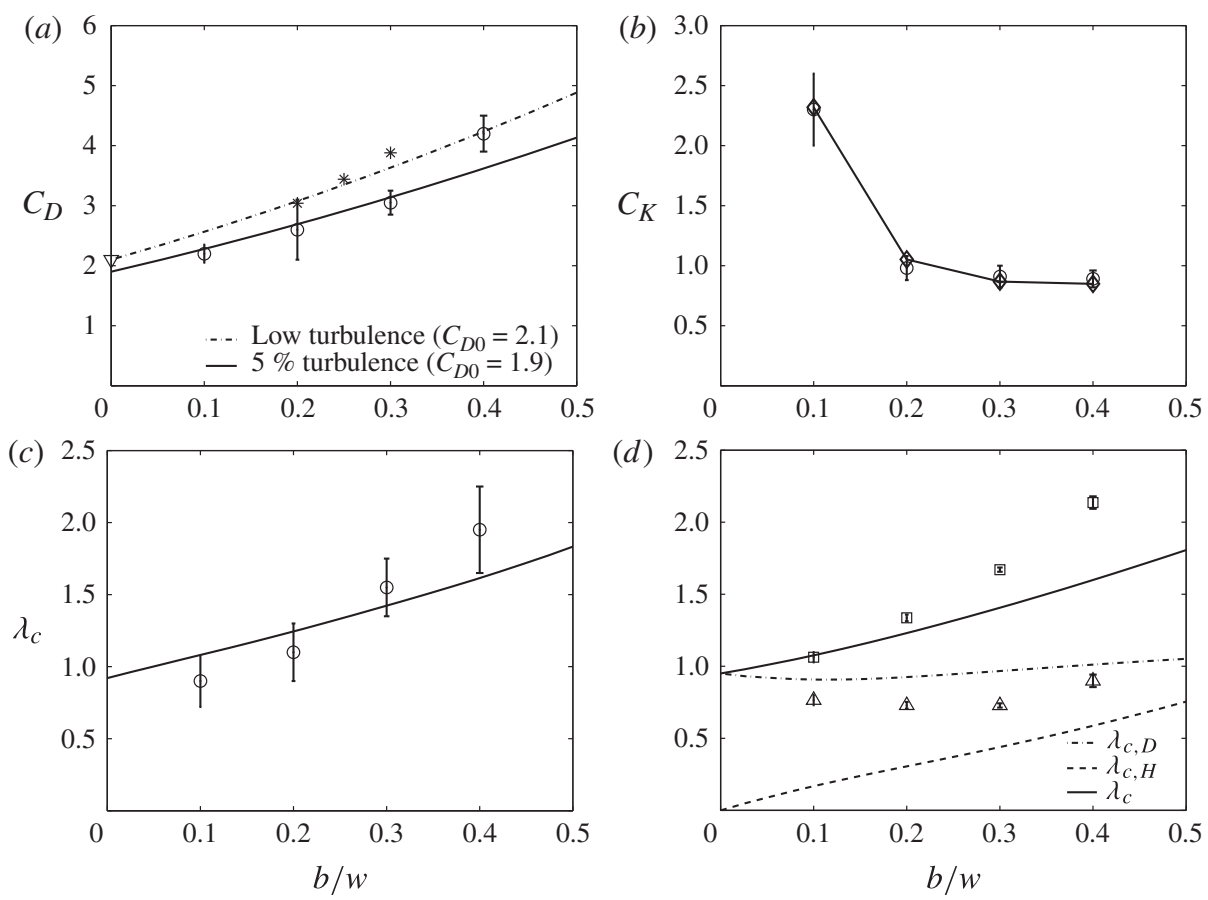

FIGURE 7. Plots as functions of the blocking $b / w$ of $(a)$ the form-drag coefficient $C_{D}$, (b) the hydrostatic-drag coefficient $C_{K}$ and $(c, d) \lambda_{c}$ for the choked flow regime. Circles represent experimental values, with error bars indicated. In $(a)$ the curves correspond to (2.8) with two different values of $C_{D 0}$, and the symbols $\nabla$ and $*$ correspond to results from Sohankar, Norberg \& Davidson (1999) and Sharify et al. (2012), respectively. In (b) the symbol $\diamond$ corresponds to estimated $C_{K}$ using measurements of $F_{1 c}, F r_{2 c}$ and $C_{D}$. In (c) the curve corresponds to (4.5). In $(d) \lambda_{c}(\square)$ is estimated using the empirical values for $C_{D}, C_{H}$ in (4.5). The form-drag and hydrostatic contributions to $\lambda_{c}$ are indicated by $\lambda_{c, D}(\triangle)$ and $\lambda_{c, H}(---)$, respectively, corresponding to the first and second terms in (4.5).

change in the depth of the water as it is swept past the obstacle, the majority of the drag is due to form drag. The fraction of the total force due to the hydrostatic component varies from 25 to $60 \%$ as the blocking fraction increases from 0.1 to 0.4 (figure $7 d$ ).

\section{Concluding remarks}

We have described an experimental study to examine the drag and lift forces on a square obstacle in a free-surface channel flow. This study is one of a few that have focused specifically on measuring and understanding the forces on the obstacles, particularly in the choked regime.

In the low-Froude-number regime, where the flow is subcritical, the flow is around the obstacle and the drag force scales as $C_{D} \rho b u_{1}^{2} h_{1} / 2$. The drag coefficient agrees with the prediction (2.8) and is consistent with studies for square cylinders in turbulent channel flows. For choked flows, the drag force scales as $C_{K} \rho b g\left(h_{1}^{2}-h_{2}^{2}\right) / 2$, and experimentally we observe that $C_{K} \simeq 1$. The experimental study was used to infer a value of $C_{H}=0.58$ for the hydrostatic coefficient. In the choked regime, the fractions of the total drag force that correspond to hydrostatic and form-drag components 
were estimated. These results tend to show that the majority of the drag, even in the choked regime, originates from form drag, with the hydrostatic drag forming a fraction less than $60 \%$ at high blocking fractions. In this regime, the total force can be estimated from $\lambda \rho b g^{1 / 3} u_{1}^{4 / 3} h_{1}^{4 / 3}$ using upstream measurements.

\section{Acknowledgements}

The Erasmus Mundus External Cooperation Window Programme financed by the European Commission is acknowledged. We thank Mr D. Mandal for designing the experimental apparatus.

\section{REFERENCES}

AwBI, H. B. 1978 Wind-tunnel-wall constraint on two-dimensional rectangular-section prisms. J. Ind. Aerodyn. 3, 285-306.

Azinfar, H. \& Kells, J. A. 2009 Flow resistance due to a single spur dike in an open channel. J. Hydraul. Res. 47, 755-763.

BenJamin, T. B. 1956 On the flow in channels when rigid obstacles are placed in the stream. J. Fluid Mech. 1, 227-248.

BetZ, A. 1925 A method for the direct determination of profile drag. Z. Flugtech. Motorluftschiffahrt 16, 42-44 (in German).

Brocchini, M. \& Peregrine, D. H. 2001 The dynamics of strong turbulence at free surfaces. Part 1. Description. J. Fluid Mech. 449, 225-254.

Fenton, J. D. 2003 The effects of obstacles on surface levels and boundary resistance in open channels. In Proceedings of the 30th IAHR World Congress (Thessaloniki, Greece, 2003) (ed. J. Ganoulis \& P. Prinos), vol. C2, pp. 9-16. Aristoteleio Panepistimio Thessalonikis.

Fenton, J. D. 2008 Obstacles in streams and their roles as hydraulic structures. In Hydraulic Structures - Proceedings of the 2nd International Junior Researcher and Engineer Workshop on Hydraulic Structures (Pisa, Italy, 30 July-1 August 2008) (ed. S. Pagliara), pp. 15-22. Pisa University Press.

Henderson, F. M. 1966 Open Channel Flow. Macmillan.

MASKEL, E. C. 1963 A theory of the blockage effects on bluff bodies and stalled wings in a closed wind tunnel. Tech. Rep. Reports and Memoranda No. 3400, Aeronautical Research Council, London.

Massey, B. S. \& Ward-Smith, J. 1998 Mechanics of Fluids. Taylor \& Francis.

NAGLER, F. A. 1918 Obstruction of bridge piers to the flow of water. Trans. ASCE 82, 334-395.

NiCOlLE, A. \& EAmES, I. 2011 Numerical study of flow through and around a circular array of cylinders. J. Fluid Mech. 679, 1-31.

Raju, K. G. R., Rana, O. P. S., Asawa, G. L. \& Pillai, A. S. N. 1983 Rational assessment of blockage effect in channel flow past smooth circular cylinders. J. Hydraul. Res. 21, 289-302.

Sharify, E. M., Saito, H., Taikan, H., Takahashi, S. \& Arai, N. 2012 Experimental and numerical study of blockage effects on flow characteristics around a square-section cylinder. In ISEM-ACEM-SEM-7th ISEM Conference, Taipei, Taiwan.

Sohankar, A., Norberg, C. \& DaVidson, L. 1999 Large eddy simulation of flow past a square cylinder. Trans. ASME J. Fluids Engng 122, 39-47.

TAmura, T. \& MiYagi, T. 1999 The effect of turbulence on aerodynamic forces on a square cylinder with various corner shapes. J. Wind Engng Ind. Aerodyn. 127, 657-662.

YARnell, D. L. 1934a Pile trestles as channel obstructions. Tech. Rep. 429, US Department of Agriculture, Washington.

YARnell, D. L. 1934b Bridge piers as channel obstructions. Tech. Rep. 442, US Department of Agriculture, Washington. 